\title{
Challenges to Improving the Chum Salmon Hatchery Program in Kitami Region, Hokkaido
}

\author{
Yasuyuki Miyakoshi
}

Kitami Salmon Enhancement Program Association, 1-5-17 Shin-machi, Abashiri, Hokkaido, 093-0046, Japan

Keywords: chum salmon, hatchery program, Hokkaido, Okhotsk Sea

In the eastern region on the Okhotsk side of Hokkaido, the Kitami Salmon Enhancement Program Association produces and releases a total of approximately 180 million chum salmon fry every year. In the last 10 years, 6,89319,144 thousand, with a mean of 12,224 thousand, chum salmon returned to this region; this quantity is equivalent to $33-48 \%$ of total return of chum salmon in Hokkaido and $28-42 \%$ in Japan. The number of chum salmon returning to the Kitami region peaked from 2003-2013 but has declined significantly since 2014. In Hokkaido, non-profit private salmon enhancement program associations established in nine regions produce and release juvenile chum salmon (Miyakoshi et al. 2013). The operating expenses of the enhancement programs are collected from fishery production; however, the recent decline in salmon catches has made the operation of the salmon enhancement associations even more difficult.

The hatchery techniques for chum salmon in Japan have been intensively developed for several decades until the 2000s (Nogawa 2010). To further raise the stocking effectiveness for chum salmon, the Kitami Salmon Enhancement Program Association continues to modify and improve hatchery procedures, which are not sticking to the conventional manual for hatchery programs. For example, at the alevin stage, fish density in the ponds is reduced and the amount of water supply is kept to a minimum, and at the fry stage, the depth of rearing ponds is deeper than the normal rearing ponds. The fish quality is checked by examining the development of gill structure repeatedly at each hatchery to evaluate the incubating and rearing conditions. Each procedure at our hatcheries is aimed to reduce stresses on fish at each life stage and improve fish quality by considering the habitat environments and ecology of chum salmon in the wild.

In addition, we are conducting our own research to improve hatchery techniques and stocking effectiveness, some of which are conducted in collaboration with national and prefectural research institutes. Specifically, buoys to observe sea water temperatures are installed in the coastal areas to decide the release timing. We are implementing releases of marked fish to evaluate the return rates by the differentiated release timing and effects of transplantation at the egg and fry stages. Furthermore, we have conducted assessments for naturally spawning chum salmon in some rivers by ourselves and have restocked chum salmon adults captured by weirs to enable them to spawn in the wild (Kitada 2014).

Because the chum salmon fishery is one of the most important ones in northern Japan, those involved in the salmon fishery and stock enhancement are eager to know the causes of the decline of chum salmon in recent years. Recent research indicates the reduction of fitness and genetic alterations by consecutive hatchery breeding of chum salmon in Japan (Kitada and Kishino 2020). Since the hatchery-based stock enhancement program has been, and will be a major management method for chum salmon in Japan, those involved in salmon fishery and stock enhancement expect that researchers will show the effective and viable measures for hatchery improvement to realize the recovery of salmon returns and a sustainable salmon fishery.

\section{REFERENCES}

Kitada, S. 2014. Japanese chum salmon stock enhancement: current perspective and future challenges. Fish. Sci. 80: 237-249.

Kitada, S., and H. Kishino. 2020. Natural and hatchery-derived selection on chum salmon: mechanisms underlying Japanese catch decline in a warming climate. bioRxiv 2020: 828780. doi: 10.1101/828780.

Nogawa, H. 2010. Development of artificial salmon propagation in Japan. J. Fish. Technol. 3: 1-8. (In Japanese with English abstract)

Miyakoshi, Y., M. Nagata, S. Kitada, and M. Kaeriyama. 2013. Historical and current hatchery programs and management of chum salmon in Hokkaido, northern Japan. Rev. Fish. Sci. 21: 469-479.

All correspondence should be addressed to Y. Miyakoshi. 